\title{
Horticultores agroecológicos em ambientes de montanha do município de Teresópolis, Rio de Janeiro
}

\section{Agroecological horticulturists in mountain environments of the municipality of Teresópolis, Rio de Janeiro, Brazil}

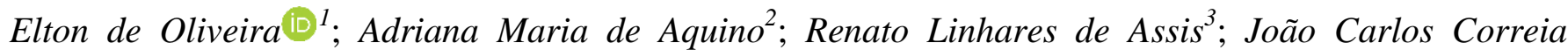 \\ Baptista Soares de Mello ${ }^{4}$.
}

${ }^{1}$ Doutor, Engenheiro Agrônomo, Universidade Federal Fluminense, Niterói, Rio de Janeiro; oliveruff2@ gmail.com; ${ }^{2}$ Doutora, Bióloga, Empresa Brasileira de Pesquisa Agropecuária, Nova Friburgo, Rio de Janeiro; adriana.aquino@embrapa.br; ${ }^{3}$ Doutor, Engenheiro Agrônomo, Empresa Brasileira de Pesquisa Agropecuária, Nova Friburgo, renato.assis@embrapa.br; ${ }^{4}$ Doutor, Engenheiro Mecânico, Universidade Federal Fluminense, Niterói, joaocsmello@ gmail.com.

\section{A R T I G O}

Recebido: 07/11/2018

Aprovado: 23/03/2019

\section{Palavras-chave:}

Agrobiodiversidade

Análise envoltória de dados

Eficiência na agricultura

Gestão da atividade agrícola

Key words:

Agrobiodiversity

Data envelopment analysis

Efficiency in agriculture

Management of agricultural activity

\section{R E S U M O}

O município de Teresópolis, na Região Serrana Fluminense se destaca na produção de hortaliças e tem toda a sua atividade agrícola em ambientes de montanha, onde a grande intensidade de cultivos provoca problemas ambientais preocupantes. As tecnologias de base agroecológica propõem ações que podem melhorar o desempenho da agricultura nesses ambientes. No entanto, para respaldar essas ações, existe necessidade de se estudar e compreender melhor a dinâmica produtiva, nesses ambientes. Este trabalho tem como objetivo analisar o desempenho de unidades produtivas (UPs) agroecológicas, usando a metodologia de análise envoltória de dados (DEA), para um estudo de caso. Foram utilizadas as variáveis de área cultivada, insumos externos, horas máquina e dias homem como inputs; faturamento e itens diferentes produzidos, como outputs do modelo DEA aplicado. A UP que apresentou o melhor desempenho produziu 34 itens diferentes, foi eficiente durante nove meses, com índice de eficiência de $96 \%$, no ano de 2017 . A combinação ou sequência entre os tipos de culturas, tamanho das áreas destinadas a cada uma e épocas de plantio, constituem uma dinâmica produtiva capaz de promover a eficiência de cada UP. A identificação das unidades eficientes e o entendimento da dinâmica e das boas práticas produtivas, pode auxiliar os agricultores com menor eficiência, na melhoria do desempenho.

\section{A B S T R A C T}

The municipality of Teresópolis, in the Rio de Janeiro mountain region, stands out in the production of vegetables, has all its agricultural activity in mountain environments, where the great intensity of crops causes disturbing environmental problems. The technologies based on agroecology propose actions that can improve the performance of agriculture in these environments. However, to support these actions, there is a need to study and better understand the productive dynamics in these environments. The objective of this work is to analyze the performance of agroecological production units (PUs), using the Data Envelopment Analysis (DEA) methodology, for a case study. The variables of cultivated area, external inputs, machine hours and man days as inputs were used; invoicing and different items produced, as outputs of the DEA model applied. The UP that presented the best performance, produced 34 different items, was efficient during nine months, with an efficiency index of $96 \%$, in the year 2017. The combination or sequence between the types of crops, size of the areas destined to each one and times of planting constitute a productive dynamic capable of promoting the efficiency of each UP. The identification of efficient units and the understanding of the dynamics and the good productive practices, can help the farmers with less efficiency, in the improvement of the performance.

\section{INTRODUÇÃO}

O Programa Agrobiodiversidade, monitoramento de
Nacional de Conservação da em seu boletim de avaliação e 2011, indicou que os sistemas agroecológicos são ambiental e socialmente mais sustentáveis e eficientes em termos de energia, e ainda apontou que a grande dificuldade na disseminação do modo de produção agroecológico é a falta de programas governamentais por 
parte do Estado (PNA, 2011). A partir de 2013 o Plano Nacional de Agroecologia e Produção Orgânica representou um grande avanço, do ponto de vista do ordenamento de ações e implementação de políticas públicas para promoção da agroecologia e da produção orgânica (PLANAPO, 2013).

Nesse contexto, o movimento de produção agroecológico promove as relações do agricultor, ambiente e sociedade; além de propor uma forma de organização que visa a conservação dos recursos naturais, a redução de insumos adquiridos fora da propriedade rural, o comércio justo, o associativismo e a manutenção da agrobiodiversidade. Considera-se que a diversidade produtiva no setor agrícola está inserida no amplo conceito de agrobiodiversidade, que pode ser definido como o conjunto de espécies e componentes da biodiversidade conservada, manejada e utilizada por agricultores (NODARI; GUERRA, 2015; MMA, 2017). A EMBRAPA (2019) define agrobiodiversidade como sendo a parte agrícola da biodiversidade, formada pelas plantas de interesse das pessoas, que, por isso, as cultivam. A agrobiodiversidade resulta do relacionamento, de milhares de anos, do ser humano com a natureza, por meio da prática de domesticação de plantas e da agricultura. A agrobiodiversidade também pode ser entendida como o processo de relações e interações da diversidade entre e dentro de espécies, dos conhecimentos tradicionais e do manejo de múltiplos agroecossistemas, sendo uma parcela da biodiversidade. Dessa forma, as interações entre as espécies cultivadas podem favorecer, além do melhor uso do espaço e da área manejada ao longo das épocas do ano, aos processos biológicos do ambiente, à ciclagem de nutrientes no solo, ao controle de pragas e doenças, e consequentemente, incrementar a eficiência do agroecossistema (GRISEL; ASSIS, 2012; NODARI; GUERRA，2015; SCHNEIDER, 2010).

Os ambientes de montanha fluminenses, conforme descrito em López Netto et al. (2017) que agregam a classificação de montanhas de Kapos et al. (2000) com a definição de ambiente em Leff (2001), são definidos como áreas com elevações variando de 300 a 1.500 metros, onde as comunidades humanas estejam presentes, levando-se em conta seus valores, ambiente natural do entorno, assim como as expressões e atividades de forma geral em determinado contexto de tempo, destacando o ponto de vista antropocêntrico dessa definição, no qual o ser humano é considerado agente essencial no contexto. Pela forma de colonização, estrutura fundiária, condições técnicas, culturais e climáticas, esses ambientes oferecem condições, para formar não somente produtos únicos e característicos, mas também para gerar uma diversidade produtiva que pode favorecer à maior eficiência e estabilidade na agricultura. Observa-se também que a maioria desses produtos são provenientes de pequenas propriedades e de agricultores familiares. Herrera et al. (2018) consideram que um dos fatores importantes para aumentar a renda, a produtividade e a diversificação, é a participação desses produtores em cooperativas agrícolas ou associações. Nota-se também que nessa última década, em especial, vem ocorrendo uma expansão do comércio de orgânicos em feiras, associações, cooperativas ou em redes, e a tendência é que aumente o número de produtores, participando diretamente em movimentos locais de produção e comercialização (DAROLT et al., 2016).
A grande intensidade da dinâmica de cultivos, aliada ao relevo e questões climáticas, vem provocando problemas ambientais preocupantes para a sustentabilidade da atividade agrícola, nos ambientes de montanha da região serrana fluminense (GRISEL; ASSIS, 2012). Em função disso, o sistema de produção agroecológico vem preconizando uma forma de organização da produção que visa a redução desses impactos negativos e melhorias das relações produtor, ambiente e consumidor. No entanto, existe uma necessidade de se estudar e compreender melhor essa dinâmica produtiva para se definir e direcionar políticas públicas específicas que promovam a sustentabilidade da agricultura nesses ambientes (GRISEL; ASSIS, 2012; NODARI; GUERRA， 2015; SCHNEIDER, 2010).

Portanto um problema, atual e relevante, consiste em definir como monitorar e avaliar o desempenho da atividade agrícola, visto que há muitos fatores que influenciam na sua eficiência produtiva. Um sistema de avaliação de eficiências pode servir de ferramenta alternativa para auxiliar a condução e aprimoramento desse processo produtivo, inclusive para auxiliar no aumento da eficiência produtiva e competitividade dos produtos orgânicos no mercado.

Pode-se verificar na literatura que o uso de DEA na agricultura tem se tornado cada vez mais usual (GOMES, 2008). Uma revisão na literatura, feita por Emrouznejad e Yang (2017), mostra um crescimento exponencial do número de publicações com teorias e aplicações de DEA em diversas áreas, nos últimos quarenta anos e destacam a sua aplicação na agricultura. Oliveira et al. $(2014 ; 2017)$ também aplicaram DEA, a partir de informações quantitativas da produção de hortaliças, para avaliação de sistemas de produção agroecológicos. A eficiência produtiva calculada com DEA foi mais eficaz na discriminação do desempenho de sistemas de cultivo, do que os indicadores agroeconômicos usualmente utilizados na literatura, segundo Bezerra Neto et al. (2007). Trabalhos como o de Salgado Junior et al. (2014) aplicaram DEA para identificar as usinas de cana-de-açúcar mais eficientes, dentre um universo de 355 no território brasileiro e classificá-las por tamanho e localização. Uma análise exploratória da eficiência produtiva, também de usinas de cana-de-açúcar, porém na região centro-sul do Brasil, utilizando DEA, foi feita por Pereira e Silveira (2016). Entretanto, nota-se uma lacuna na literatura, onde pode-se perceber poucos trabalhos que contemplem a diversidade produtiva na eficiência de unidades de produção agrícolas.

O objetivo desse estudo foi avaliar o desempenho de um conjunto de agricultores orgânicos de base agroecológica. Analisando o comportamento dos índices de eficiência de suas Unidades Produtivas (UPs), ao longo dos meses do ano de 2017. Considerando não somente o aspecto econômico, mas também a diversidade de cultivos como indicadora da agrobiodiversidade e ressaltando a dinâmica produtiva na promoção das melhores práticas dentro do manejo desses agrossistemas.

\section{MATERIAL E MÉTODOS}

Este trabalho trata-se de um estudo de caso, baseado na análise de dados quantitativos de produção, coletados de forma primária, durante os doze meses do ano de 2017, junto a nove Unidades Produtivas (UPs) de horticultura orgânica, vinculadas à Associação Agroecológica de Teresópolis (AAT) que congrega agricultores submetidos ao Sistema 
Participativo de Garantia (SPG) da conformidade orgânica e é filiada à Associação de Agricultores Biológicos do Estado do Rio de Janeiro (ABIO). Todas essas nove UPs pertencem ao município de Teresópolis que está localizado na Região Serrana e tem posição destacada na produção agrícola fluminense. Este município apresentou o segundo maior faturamento com agricultura do estado do Rio de Janeiro, no ano de 2017, foram $\mathrm{R} \$ 1$ 197.059.870,00, sobressaindo-se na produção de hortaliças do grupo das folhosas como alface, couve, salsa e brócolos (EMATER-RIO, 2017). A sede municipal apresenta elevação de 871 metros e coordenadas geográficas com $22^{\circ} 24^{\prime} 44^{\prime \prime}$ de latitude sul e $42^{\circ} 57^{\prime} 56^{\prime \prime}$ de longitude oeste (IBGE, 2015), e toda a sua atividade agrícola é desenvolvida em ambientes de montanha.

Considerando um conjunto de agricultores que possuem características semelhantes, é razoável supor que eles poderão ter diferentes resultados, dado que praticam diferentes estratégias produtivas, com diferentes intensidades no uso de insumos, e consequentes variações nas quantidades de produtos, afetando os desempenhos de cada Unidade Produtiva (UP). Neste sentido, a eficiência produtiva pode ser avaliada considerando-se variáveis que representem a preocupação de cada produtor em melhorar suas práticas de conservação e manejo de seu agrossistema.

A forma, os itens e os tipos dos dados para coleta, foram definidos de forma participativa, procurando identificar os aspectos mais relevantes; considerando as dificuldades e sugestões do produtor em quantificar, armazenar e disponibilizar informações periódicas da sua propriedade, para serem abordados e analisados de acordo com o objetivo deste estudo. Este levantamento foi efetuado a partir de um formulário, aplicado em cada UP, durante os doze meses do ano de 2017, completando um total de 108 observações, onde especificou-se o tipo, a quantidade e o valor dos itens considerados, que serviram de componentes para as seis variáveis utilizadas na análise: área cultivada, insumos externos totais, horas máquina, dias homem, faturamento total em reais e número de itens.

A área cultivada (AC), se refere à área manejada para produção agrícola de todas as culturas vegetais, dentro de cada UP. Em função da dinâmica local das rotações de cultivo de hortaliças essa área pode variar a cada mês, dentro de uma mesma UP. Considera-se que o produtor procura aproveitar da melhor maneira possível cada espaço de AC em sua propriedade. Portanto, esta variável consiste em um recurso fundamental na atividade agrícola.

Os insumos externos totais (IE) considerados, foram o somatório do valor em reais do consumo de: energia elétrica; consumo de combustíveis para transporte, comercialização e acionamento de bombas de irrigação; consumo com sementes, mudas, adubos e caldas fitossanitárias. Considera-se que todos esses insumos podem ser minimizados quando aplicados os princípios agroecológicos preconizados por essas UPs analisadas.

As horas máquina (HM) foram referentes ao somatório das horas de uso de roçadeira, trator e micro trator. Principalmente do ponto de vista da conservação de solos, a redução da intensidade dessa mecanização pode diminuir as perdas dos atributos físicos e químicos ligados à fertilidade de solos, o que é desejável.

Dias homem $(\mathrm{DH})$ foram referentes ao somatório dos dias de trabalho de familiares e diarista contratados. Os dias de trabalho da família do agricultor e dos contratados devem ser valorizados e otimizados. Consequentemente, é importante controlar essas horas de mão de obra, consideradas como recurso.

O faturamento total em reais (FT) foi obtido pelo somatório dos produtos de cada item produzido pelo seu preço de comercialização. Essa variável reflete o caráter econômico da atividade.

O número de itens (NI) foi referente a quantidade total de itens diferentes produzidos nos diversos cultivos da DMU observada. A maior diversidade pode contribuir para a otimização de uso do espaço e eficiência produtiva na agricultura, aumentando a robustez da atividade, dentro dos princípios agroecológicos, contribuindo para a agrobiodiversidade.

A metodologia de Análise Envoltória de Dados - DEA (do inglês, Data Envelopment Analysis) (CHARNES et al., 1978) foi escolhida para a avaliação de desempenho realizada neste estudo. DEA usa o conceito de eficiência, definido por Farrel (1957) como a máxima razão entre uma soma ponderada dos produtos e uma soma ponderada dos recursos, para fazer uma análise comparativa entre as eficiências de um conjunto de unidades produtivas ou unidades tomadoras de decisão, entretanto, DEA não é uma função de produção (COOK et al., 2014). Funcionando como uma ferramenta para medida de eficiência, esta abordagem é capaz de incorporar diversos recursos e produtos para o cálculo de índices de eficiência.

Foi imprescindível, no entanto, considerar conforme levantado por Dyson et al. (2001) e Cook et al. (2014), as premissas e restrições metodológicas de DEA, nas escolhas e no uso dos tipos, quantidades e proporções entre o número de variáveis e o número de DMUs utilizadas. Esses cuidados nessa etapa preliminar de planejamento e definições da modelagem DEA, foram importantes para não provocar distorções e/ou interpretações incorretas dos resultados gerados. Lembra-se que na modelagem $D E A$, as unidades produtivas são chamadas $D M U s$, os recursos são chamados de inputs e os produtos de outputs.

Os dados coletados foram consolidados em seis variáveis, sendo quatro consideradas como recursos e duas como produtos, para aplicação do modelo DEA. Portanto, área cultivada, insumos externos totais, horas máquina e dias homem foram os inputs; faturamento e itens diferentes produzidos, os outputs do modelo DEA aplicado.

Cada uma das 108 observações efetuadas constituiu uma DMU, formada pela combinação da unidade produtiva/mês (UP/mês), ou seja, 01/jan é uma DMU diferente de 01/fev, conforme utilizado em Soares de Mello et al., (2003) e Oliveira et al. (2014). Esta combinação permitiu comparar o desempenho de cada UP com ela mesma e com as demais, ao longo dos meses analisados. Possibilitando assim, fazer um acompanhamento do comportamento das eficiências de cada UP durante uma série temporal dos 12 meses do ano de 2017.

O modelo DEA escolhido foi o clássico BCC, considerando-se que não há certeza de que o aumento dos inputs acarrete um aumento proporcional dos outputs, ou seja, pode haver retornos de escala variáveis. Essa característica permite que unidades produtivas de tamanhos ou áreas cultivadas diferentes, sejam analisadas conjuntamente. A orientação do modelo foi para outputs, pois pretende-se aumentar os outputs mantendo-se os inputs constantes. Ou seja, ele compara o que foi produzido (outputs) com o que poderia ter sido produzido com os mesmos recursos (inputs). 
Maiores detalhes dessa metodologia podem ser vistos em (BANKER et al., 1984). Este Problema de Programação Linear (PPL) gera a equação 1 .

Min $h_{\circ}=\sum_{i} v_{i} x_{i o}+v_{*}$

Sujeito a:

$\sum_{j} u_{j} y_{j o}=1$

$\sum_{j} u_{j} y_{j k}-\sum_{i} v_{i} x_{i k}-v_{*} \leq 0, \forall k$

$u_{j} \geq 0, v_{i} \geq 0, \forall j, i$

$v_{*} \in \mathrm{R}$

Em que: $\boldsymbol{h}_{\circ}$ - eficiência da DMU em análise; $\boldsymbol{x}_{\boldsymbol{i} \boldsymbol{o}}$ - os inputs; $\boldsymbol{y}_{\boldsymbol{j} \boldsymbol{o}}$ - os outputs; $\boldsymbol{v}_{\boldsymbol{i}}$ e $\boldsymbol{u}_{\boldsymbol{j}}$ - pesos calculados pelo modelo para os respectivos inputs e outputs; $v_{*}$ - fator de escala.

A partir da aplicação desse modelo, foram obtidos os índices de eficiência para cada DMU. Esses índices revelados podem variar de 0 a 1 , sendo 1 eficientes e zero, ineficiente. Utilizou-se o programa Sistema Integrado de Apoio à Decisão - SIAD, para execução dos cálculos dos índices considerados nesta análise DEA (ANGULO-MEZA et al., 2005).
O conceito de eficiência na produção agrícola aplicado foi relativo às variáveis consideradas, ou seja, aos recursos e produtos incorporados e contemplados no processo produtivo observado. A metodologia DEA foi baseada em valores extremos e caracterizada pela não utilização de inferências estatísticas, de medidas de tendência central ou de aproximações paramétricas, portanto, não tem caráter estocástico, não sendo necessários os cuidados habituais da estatística relativo ao tamanho de amostras. Foi ainda invariante quanto a escala de suas variáveis, por isso, cada variável foi incluída no modelo com sua unidade de medida de escala original, sem transformações para uniformizações de escala (CHARNES et al., 1994; COOPER et al., 2000). Em DEA, o efeito ou representatividade da análise se encerra no conjunto de DMUs analisado. Ou seja, o resultado desta, pode mudar com alterações no conjunto de DMUs considerado (COOPER et al., 2007). Sendo, com isso, os resultados apresentados, válidos para considerações entre as unidades participantes ou observadas, não permitindo a extrapolação de suas conclusões, que estão restritas às unidades produtivas e às variáveis em análise.

\section{RESULTADOS E DISCUSSÃO}

Dentro do conjunto das UPs analisadas, o tamanho das áreas cultivadas variou de 0,8 a 7 hectares e o faturamento anual de $\mathrm{R} \$ 12.059,00$ a $\mathrm{R} \$ 112.057,50$ (Tabela 1$)$.

Tabela 1. Valores acumulados das variáveis consideradas, ao longo de todo o ano de 2017, por cada Unidade Produtiva (UP) de horticultura orgânica, vinculadas à Associação Agroecológica de Teresópolis, Rio de Janeiro.

\begin{tabular}{|c|c|c|c|c|c|c|c|c|}
\hline \multirow{2}{*}{ UPs } & \multicolumn{3}{|c|}{ Área Cultivada (ha) } & \multirow{2}{*}{ Insumos Externos totais $\mathrm{R} \$$} & \multirow{2}{*}{$\mathrm{HM}$} & \multirow{2}{*}{$\mathrm{DH}$} & \multirow{2}{*}{ NIDPa } & \multirow{2}{*}{ Faturamento ( $\mathrm{R} \$$ /ano) } \\
\hline & $\min$ & $\max$ & med & & & & & \\
\hline UP2 & 0,80 & 1,10 & 0,90 & $6.351,50$ & 124,5 & 511 & 45 & $14.785,00$ \\
\hline UP3 & 2,00 & 3,30 & 2,46 & $7.451,15$ & 66,7 & 563 & 48 & $22.374,80$ \\
\hline UP5 & 4,00 & 6,00 & 4,17 & $5.733,44$ & 130,0 & 475 & 31 & $35.700,00$ \\
\hline UP7 & 3,00 & 4,65 & 4,32 & $18.444,00$ & 129,3 & 1479 & 56 & $112.057,50$ \\
\hline UP8 & 0,90 & 0,90 & 0,90 & $10.449,00$ & 29,0 & 311 & 34 & $42.675,50$ \\
\hline UP9 & 1,00 & 1,00 & 1,00 & $6.228,00$ & 17,5 & 406 & 26 & $12.059,00$ \\
\hline UP10 & 4,00 & 5,00 & 4,42 & $21.225,00$ & 41,0 & 613 & 35 & $103.205,00$ \\
\hline UP11 & 2,00 & 2,00 & 2,00 & $8.073,00$ & 88,0 & 878 & 47 & $50.866,00$ \\
\hline UP12 & 3,00 & 7,00 & 5,25 & $26.776,00$ & 131,0 & 899 & 49 & $101.658,00$ \\
\hline
\end{tabular}

HM - Horas Máquina, DH - Dias Homem, NIDPa - Número de Itens Diferentes Produzidos no ano

Assim como considerado nos trabalhos de Machado et al. (2016) e Wu et al. (2009), as UPs referentes às DMUs com melhores desempenhos podem ser indicadas como referência para as unidades ineficientes deste estudo. Identificou-se as UPs 8, 2 e 12, como as de maior número de meses eficientes e com os maiores índices de eficiências médias no ano (Tabela 2).

Março foi o mês com o maior número de DMUs eficientes (5), a maior eficiência média $(92,9 \%)$ e também o mais estável, com o menor DP $(0,11)$ (Tabela 2$)$.

Verifica-se que a UP 8 foi eficiente em 9 meses e produziu 34 itens diferentes, tendo índice de eficiência média de $96 \%$ com desvio padrão igual a 0,09 , tendo o melhor desempenho e estabilidade de todo o conjunto (Tabela 1 e 2). Nesta UP, a reforma de canteiros no mês de junho/2017, foi o principal motivo da redução da eficiência atingindo o índice de $69 \%$. A safra de raízes (aipim) e tubérculos (batata doce, inhame) manteve a receita, a recuperação da eficiência ocorreu já no mês seguinte, julho/2017, com a safra de folhosas. Essa prática e o seu resultado, revela sua estratégia produtiva como referência para este conjunto de produtores.

Embora a UP 2 tenha produzido uma quantidade maior de itens diferentes (45) que a UP 8, em uma mesma área cultivada média, foi superada por esta UP, tanto no índice médio de eficiência como no número de meses eficientes, no ano (Tabelas 1 e 2). Nota-se também que a UP 2, ao reduzir a aquisição de insumos e aumentar a diversidade de itens, houve um aumento de horas máquina e dias homem, tornando-a eficiente durante somente seis meses no ano.

Observa-se que a UP 12 foi eficiente durante cinco meses do ano e apresentou média dos índices de eficiência de 87\% (Tabela 2). Esta UP expandiu sua área cultivada, de 3 para $7 \mathrm{ha}$; proporcionalmente, essas expansões provocam baixas de eficiência, pois a área adicionada, tida como 
recurso, pode levar meses para gerar aumento na quantidade de produtos. Este foi o principal motivo das reduções dos seus índices nos meses de fevereiro, julho e agosto. Mesmo assim, conseguiu ser eficiente no mês de dezembro, totalizando cinco meses eficientes, e fechou o ano com a maior área cultivada do conjunto e 49 itens diferentes produzidos (Tabela 1 e 2). Este resultado mostra que este agricultor soube manejar bem seus novos plantios, nas expansões da área cultivada dentro da propriedade.

Tabela 2. Índices de eficiência das unidades produtivas (UPs) de horticultura orgânica, vinculadas à Associação Agroecológica de Teresópolis, Rio de Janeiro, ao longo dos meses de 2017.

\begin{tabular}{cccccccccccccccccc}
\hline UPs & JAN & FEV & MAR & ABR & MAI & JUN & JUL & AGO & SET & OUT & NOV & DEZ & MED & MAX & MIN & DP & NME \\
\hline 8 & 1,00 & 1,00 & 1,00 & 1,00 & 1,00 & 0,69 & 1,00 & 1,00 & 0,94 & 1,00 & 1,00 & 0,89 & 0,96 & 1,00 & 0,69 & 0,09 & 9 \\
2 & 1,00 & 1,00 & 1,00 & 0,94 & 0,80 & 0,80 & 1,00 & 1,00 & 1,00 & 0,79 & 0,92 & 0,72 & 0,91 & 1,00 & 0,72 & 0,11 & 6 \\
12 & 1,00 & 0,72 & 1,00 & 1,00 & 0,78 & 0,97 & 0,73 & 0,62 & 1,00 & 0,83 & 0,85 & 1,00 & 0,87 & 1,00 & 0,62 & 0,14 & 5 \\
10 & 0,61 & 0,81 & 0,99 & 0,72 & 0,74 & 1,00 & 0,78 & 1,00 & 0,80 & 0,97 & 1,00 & 1,00 & 0,87 & 1,00 & 0,61 & 0,14 & 4 \\
11 & 0,75 & 0,68 & 0,78 & 0,91 & 1,00 & 0,92 & 0,86 & 0,86 & 0,84 & 0,63 & 0,88 & 1,00 & 0,84 & 1,00 & 0,63 & 0,12 & 2 \\
7 & 0,79 & 0,69 & 0,84 & 0,66 & 0,92 & 1,00 & 1,00 & 0,88 & 0,74 & 0,82 & 1,00 & 0,86 & 0,85 & 1,00 & 0,66 & 0,12 & 3 \\
5 & 1,00 & 0,79 & 1,00 & 1,00 & 0,75 & 0,93 & 0,74 & 0,58 & 0,58 & 0,63 & 0,73 & 0,92 & 0,80 & 1,00 & 0,58 & 0,16 & 3 \\
3 & 0,57 & 0,76 & 1,00 & 0,66 & 0,57 & 0,84 & 1,00 & 1,00 & 1,00 & 0,67 & 0,88 & 0,80 & 0,81 & 1,00 & 0,57 & 0,17 & 4 \\
9 & 0,42 & 1,00 & 0,75 & 0,41 & 0,47 & 0,47 & 0,35 & 0,32 & 0,34 & 1,00 & 0,74 & 0,42 & 0,56 & 1,00 & 0,32 & 0,25 & 2 \\
\hline Média & 0,79 & 0,83 & 0,93 & 0,81 & 0,78 & 0,85 & 0,83 & 0,81 & 0,81 & 0,81 & 0,89 & 0,85 & & & & & \\
MAX & 1,00 & 1,00 & 1,00 & 1,00 & 1,00 & 1,00 & 1,00 & 1,00 & 1,00 & 1,00 & 1,00 & 1,00 & & & & & \\
MIN & 0,42 & 0,68 & 0,75 & 0,41 & 0,47 & 0,47 & 0,35 & 0,32 & 0,34 & 0,63 & 0,73 & 0,42 & & & & & \\
DP & 0,22 & 0,14 & 0,11 & 0,21 & 0,18 & 0,17 & 0,21 & 0,25 & 0,22 & 0,15 & 0,11 & 0,19 & & & & & \\
NEM & 4 & 3 & 5 & 3 & 2 & 2 & 4 & 4 & 3 & 2 & 3 & 3 & & & & & \\
\hline
\end{tabular}

MAX - Máximo. MIN - Mínimo. MED - Média. DP - Desvio Padrão. NME - Número de Meses Eficientes. NEM - Número de UPs Eficientes no Mês.

A UP 7 gerou o maior número de itens diferentes produzidos no ano (56). Porém, apresentou eficiência média de $85 \%$, sendo eficiente por três meses. Percebe-se que sua área cultivada esteve em expansão, o que pode ter ocasionado este desempenho menor em sua fase inicial, já que o aumento do recurso área cultivada pode não elevar imediatamente as quantidades de produtos, na agricultura (Tabelas 1 e 2).

A UP 9 foi eficiente por dois meses, apresentou índice de eficiência médio de 56\% (Tabela 2), o mais baixo do conjunto e manteve sua área cultivada sem alterações significativas durante todo o ano (Tabela 1).

Verifica-se um bom desempenho da UP 10 que foi eficiente por quatro meses, com $87 \%$ de índice de eficiência, considerando-se que aumentou de 4 para 5 ha de área cultivada e produziu 35 itens diferentes, no ano (Tabelas 1 e 2).

No trabalho de Pereira et al. (2016) também foi verificado, assim como nesse estudo, que as unidades de produção orgânicas de base agroecológica nesses ambientes, apresentam maior diversidade produtiva, maior domínio sobre diversas formas de comercialização e gestão. Assis et al. (2016) enumeram e destacam, entre os preceitos da agroecologia, a diversificação de cultivos e a menor dependência possível de insumos externos. O manejo agroecológico utiliza estratégias que podem tornar a agricultura mais eficiente e sustentável, entre elas o manejo da agrobiodiversidade (GRISEL; ASSIS, 2012; NODARI; GUERRA, 2015).

A dinâmica produtiva, descrita no trabalho de Grisel e Assis (2015), está sendo identificada na sua forma eficiente, pela análise efetuada neste estudo. Essa dinâmica produtiva, varia de uma UP para outra e faz parte de uma estratégia de produção formada pela percepção individual do agricultor.
Dinâmica que interferiu nas seis variáveis consideradas no cálculo da eficiência avaliada e faz parte de importantes decisões, por parte de cada produtor. Esta análise temporal das DMUs auxilia a demonstrar melhor seus desempenhos, possibilitando uma visão mais detalhada da realidade das unidades de produção agrícola. Este monitoramento de cada DMU ao longo do tempo poderá auxiliar no planejamento e incremento da eficiência na atividade agrícola.

Constata-se, no conjunto de DMUs analisado, que o NIDP, variou no mês de 5 a 33, e no ano de 26 a 67 . Entre as DMUs eficientes, este NIDP mensal, variou de 7 a 33, e de 31 a 67 durante todo o ano de 2017 (Tabelas 1 e 2). O comportamento que as UPs agroecológicas apresentam com a estratégia de diversificação produtiva, em maior ou menor intensidade, é justificado pelos benefícios que as interações entre as culturas propiciam na qualidade da produção e na eficiência produtiva (GRISEL; ASSIS, 2012; NODARI; GUERRA, 2015; SCHNEIDER, 2010), e pela intenção de atender, motivar e despertar o desejo de consumo dos seus fregueses e retê-los, com a maior gama possível de produtos, sabores e experiências alimentares (DAROLT et al., 2016).

Portanto, o resultado das combinações ou sequências entre os tipos de culturas e suas respectivas quantidades produzidas, interferiu na eficiência dessas unidades produtivas, durante os meses do ano, sendo praticável atingir a eficiência com muitas dessas combinações.

Assim como no trabalho de Abreu e Watanabe (2016), procurou-se com esse estudo, chamar a atenção para o espaço que a inovação, baseada na diversificação agroecológica, pode propiciar nas atividades dos sistemas de produção agrícolas que buscam mais eficiência e sustentabilidade.

Observa-se que, dentro das UPs analisadas, o dimensionamento das áreas de plantio de cada cultura precisa 
ser feito com um escalonamento, para dosar a colheita que será comercializada semanalmente, durante cada mês. Isso porque, os itens produzidos não podem ser estocados, pois são perecíveis. Sem esse cuidado, o produtor pode aumentar suas perdas por falta de demanda, ou ter que baixar seus preços de comercialização, para tentar escoar o excedente produzido. Além disso, alguns itens tem a produção comprometida em função das estações do ano, e precisam ser rotacionados, com outros itens propícios em determinadas épocas do ano. Existem também os itens que podem ser produzidos durante todos os meses do ano, com uma oferta constante. Dessa forma, este trabalho contribui para a gestão e o planejamento da propriedade que, de acordo com Darolt et al. (2016) p. 14, são essenciais para o desenvolvimento e organização desses sistemas de produção.

Nessa análise efetuada, fundamentada na comparação conjunta de unidades de produção agrícolas, como os resultados são obtidos através de observações reais, as metas que podem ser estipuladas, ou alvos a serem atingidos afim de tornar estes produtores eficientes, baseados nas UPs com melhores desempenhos, são cabíveis de serem alcançados (GOMES et al., 2009).

Portanto, conforme Santos e Cândido (2013) enfatizam, essa avaliação de desempenho efetuada no presente estudo, também busca auxiliar na melhoria da gestão da atividade agrícola, possibilitando um estudo comparativo entre os desempenhos dos produtores rurais da região observada, dando respaldo para ações conjuntas, principalmente quando se pretende ressaltar o propósito do associativismo.

Desta forma, essa discussão também contribui, conforme conclusões dos trabalhos de Herrera et al. (2018); Nodari e Guerra (2015), para promover a aglutinação de duas estratégias. Onde, a primeira considera que a organização em associações e através de iniciativas conjuntas, o produtor pode aprimorar seu desempenho; e a segunda de que a diversidade produtiva pode promover a eficiência dos sistemas agrícolas sustentáveis.

Esse estudo, também, pode auxiliar o trabalho do gestor das unidades produtivas agroecológicas onde, conforme destacam Gemma et al., 2010, devido à grande variedade de produtos oferecidos, exigem a necessidade de expertise no trato com as diferentes espécies vegetais e suas integrações com os demais sistemas de produção. Por meio do desenvolvimento e da conexão de variados saberes e competências, este gestor pode elaborar estratégias para superar as diversas dificuldades relacionadas com os aspectos tecnológicos, financeiros, mercadológicos, ambientais e humanos da sua produção.

As interações entre as culturas agrícolas dentro da mesma propriedade podem otimizar o uso do espaço, da mão de obra, das máquinas e de insumos, aumentando a eficiência e promovendo a sua estabilidade ao longo do tempo. Porém, o maior número de itens produzidos ou maior número de culturas diferentes em si só, não garante este resultado. A combinação ou sequência entre os tipos de culturas, tamanho das áreas destinadas a cada uma e épocas de plantio, constituem uma dinâmica produtiva capaz de promover a eficiência de cada UP.

Em trabalhos futuros, para a análise de um conjunto maior e menos homogêneo de UPs, sugere-se a divisão em grupos de similaridade de atividades, como de produção animal ou processamento de alimentos integrados à pratica agrícola, aplicando modelos DEA diferentes, em cada grupo.
Outra possibilidade na aplicação do modelo proposto nesse estudo, seria para uma complementação do trabalho de Gazzola et al., 2018 que fizeram uma comparação descritiva entre estabelecimentos de produção agropecuária orgânica e convencional, com dados secundários. Sugere-se, para trabalhos futuros, uma análise comparativa entre as eficiências de unidades produtivas orgânicas e convencionais, tomando-se as mesmas variáveis aqui consideradas.

\section{CONCLUSÃo}

A aplicação do sistema de Análise Envoltória de Dados permite a identificação e reconhecimento das unidades produtivas com as melhores práticas, valorizando o trabalho dos agricultores com as melhores estratégias de produção. A diversidade produtiva, representa a agrobiodiversidade, e propicia estabilidade e incremento dos índices de eficiência revelados.

A expansão das áreas cultivadas interfere nas variações dos níveis de eficiência das UPs, ao longo dos meses do ano. Portanto, a estabilização do tamanho da área cultivada total da UP, ao longo do ano, contribui para efetivar os índices de eficiência produtiva.

A identificação das UPs com melhores desempenhos é importante para o conhecimento e a compreensão de suas dinâmicas produtivas, podendo auxiliar no planejamento dos agricultores que buscam enxergar melhor as oportunidades e as dificuldades nas relações entre produção, ambiente e comercialização.

\section{AGRADECIMENTOS}

AAT (Associação Agroecológica de Teresópolis), CAPES, CNPq, EMBRAPA, UFF, UFRRJ e ao PARNASO (Parque Nacional da Serra dos Órgãos) sede Teresópolis, pelo apoio à pesquisa e ao trabalho de campo.

\section{REFERÊNCIAS}

ABREU, L. S.; WATANABE, M. A. Agricultores familiares do Sul da Amazônia: Desafios e estratégias para inovação agroecológica de sistemas de produção. Revista Verde de Agroecologia e Desenvolvimento Sustentável, v. 11, n. 5, p. 114-122, 2016. http://dx.doi.org/10.18378/rvads.v11i5.4202

ANGULO MEZA, L.; BIONDI NETO, L.; SOARES DE MELLO, J. C. C. B.; GOMES, E. G. ISYDS-Integrated System for Decision Support (SIAD-Sistema Integrado de Apoio à Decisão): a software package for data envelopment analysis model. Pesquisa Operacional, n. 25, p. 493-503, 2005.

ASSIS, R. L.; ARAÚJO, J. S. P.; ANJOS, L. H. C.; AQUINO, A. M. Agroecologia, produção orgânica e agricultura urbana no Rio de Janeiro. Boletim informativo da Sociedade Brasileira de Ciência do Solo, v. 42, n. 3, p.32-35, 2016.

BANKER, R. D.; CHARNES, A.; COOPER, W. W. Some models for estimating technical scale inefficiencies in Data Envelopment Analysis. Management Science, v. 30, n. 9, p.1078-1092, 1984. 
BEZERRA NETO, F.; GOMES, E. G.; OLIVEIRA, A. M. Produtividade biológica em sistemas consorciados de cenoura e alface avaliada através de indicadores agroeconômicos e métodos multicritério. Horticultura Brasileira, n.25, p.193198, 2007.

CHARNES, A.; COOPER, W. W.; RHODES, E. Measuring the efficiency of decision-making units. European Journal of Operational Research, Amsterdam, v. 2, n. 3, p.429-444, 1978.

CHARNES, A.; COOPER, W. W.; LEWIN, A. Y.; SEIFORD, L. M. Data Envelopment Analysis: theory, methodology and applications. Nor well, Massachusets, EUA: Kluwer Academic Publishers, 1994. 512 p.

COOK, W. D.; TONE, K.; ZHU, J. Data envelopment analysis: Prior to choosing a model. Omega, n. 44, p.1-4, 2014.

COOPER, W. W.; SEIFORD, L. M.; TONE, K. Data Envelopment Analysis: a comprehensive text with models, applications, references and DEA-solver software. Norwell, Massachusets, EUA: Kluwer Academic Publishers, 2000. 307 p.

COOPER, W. W.; SEIFORD, L. M.; TONE, K. Data Envelopment Analysis - A comprehensive text with models, applications, references and DEA-solver software. Springer Science, 2007. 512 p.

DAROLT, M. R.; LAMINE, C.; BRANDENBURG, A.; ALENCAR, M. C. F.; ABREU, L. S. Alternative food networks and new producer-consumer relations in France and in Brazil. Ambiente \& Sociedade, v. 19, n. 2, p. 1-22, 2016. http://dx.doi.org/10.1590/1809-4422ASOC121132V1922016

DYSON, R. G.; ALLEN R.; CAMANHO A. S.; PODINOVSKI, V. V.; SARRICO C. S.; SHALE, E. A. Pitfalls and protocols in DEA. European Journal of Operational Research, n. 132, p. 245-259, 2001.

EMATER-RIO - Empresa de Assistência Técnica e Extenção Rural do Estado do Rio de Janeiro. Relatório de Acompanhamento Sistemático da Produção Agrícola ASPA, 2017. Disponível em < http://www.emater.rj.gov.br/images/munic2017.htm>. Acesso em: 07 abr. 2019.

EMBRAPA, Empresa Brasileira de Pesquisa Agropecuária. Conceito de Agrobiodiversidade, 2019. Disponível em: <https://www.embrapa.br/contando-ciencia/agricultura//asset_publisher/FcDEMJIbvFle/content/o-que-eagrobiodiversidade-/1355746?inheritRedirect=false $>$. Acesso em: 21fev 2019.

EMROUZNEJAD, A.; YANG, G-L. A survey and analysis of the first 40 years of scholarly literature in DEA: 1978-2016, Socio-Economic Planning Sciences, 2017. http://dx.doi.org/10.1016/j.seps.2017.01.008
FARREL, M. J. The measurement of productive efficiency. Journal of the Royal Statistic Society, series A, part 3, p. 253290, 1957.

GAZZOLA, R.; GOMES, E. G.; SOUZA, G. S.; WANDER, A. E. Agricultura orgânica e convencional em Santa Catarina: Análise comparativa dos produtores. Revista Verde de Agroecologia e Desenvolvimento Sustentável, v.13, n. 1, p. 89-96, 2018. http://dx.doi.org/10.18378/rvads.v13i1.4233

GEMMA, S. F. B.; TERESO, M. J. A.; ABRAHÃO, R. F. Ergonomia e complexidade: o trabalho do gestor na agricultura orgânica na região de Campinas - SP. Ciência Rural, v. 40, n. 2, p. 318-324, 2010.

GOMES, E. G. Uso de modelos DEA em agricultura: Revisão da literatura. Engevista, n. 10, p. 27-51, 2008.

GOMES, E. G.; SOARES DE MELLO, J. C. C. B.; MANGABEIRA, J. A. C. Estudo da sustentabilidade agrícola em município amazônico com análise envoltória de dados. Pesquisa Operacional, v. 29, n. 1, p. 23-42, 2009.

GRISEL, P. N.; ASSIS, R. L. Adoção de Práticas Agrícolas Sustentáveis: Estudo de Caso de um Sistema de Produção Hortícola Familiar em Ambiente de Montanha. Cadernos de Ciência \& Tecnologia, Brasília, v. 29, n. 1, p. 133-158, 2012.

GRISEL, P. N.; ASSIS, R. L. Dinâmica agrária da Região Sudoeste do município de Nova Friburgo e os atuais desafios de sua produção hortícola familiar. Seropédica: Embrapa Agrobiologia. Documentos, 299, 2015. 83 p.

HERRERA, G. P.; LOURIVAL, R.; COSTA, R. B.; MENDES, D. R. F.; MOREIRA, T. B. S.; ABREU, U. G. P.; CONSTANTINO, M. Econometric analysis of income, productivity and diversification among smallholders in Brazil. Land Use Policy, 2018. https://doi.org/10.1016/j.landusepol.2018.02.025

IBGE, Instituto Brasileiro de Geografia e Estatística. Altitude e coordenadas geográficas das sedes municipais, segundo as Regiões de Governo e municípios do Estado do Rio de Janeiro, 2015. Disponível em <http://www.ceperj.rj.gov.br/ceep/info territorios/posicao_ex tencao.html>. Acesso em 04 abr. 2017.

KAPOS, V.; RHIND, J.; EDWARDS, M.; PRICE, M. F.; RAVILIOUS, C. Defining mountains by topography only. 2000. In: Millenium Ecosystem Assessment. Ecosystems and human wellbeing: Mountain Systems. Washington, DC: Island Press and World Resources Institute, 2005. Disponível em:

<https://www.millenniumassessment.org/documents/docume nt.293.aspx.pdf>. Acesso em: 19 fev. 2019.

LEFF, E. Saber ambiental: sustentabilidade, racionalidade, complexidade, poder. Petrópolis: Vozes, 2001.

LÓPEZ NETTO, A.; ASSIS, R. L.; AQUINO, A. M. Ações Públicas para o Desenvolvimento Rural Sustentável dos 
Ambientes de Montanha Brasileiros. Desenvolvimento em Questão, v. 15, n. 39, p. 141-170, 2017.

MACHADO, L. G.; SOARES DE MELLO, J. C. C. B.; ROBOREDO, M. C. Efficiency Evaluation of Brazilian Electrical Distributors Using DEA Game and Cluster Analysis. IEEE Latin America Transactions, v. 14, n. 11, p. 4499-4505, 2016.

NODARI, R. O.; GUERRA, M. P. A agroecologia: estratégias de pesquisa e valores. Estudos Avançados, v. 29, n.83, p. 183-207, 2015.

OLIVEIRA, E.; ANDRADE， F. V. S.; SOARES DE MELLO, J. C. C. B.; MACHADO, T. B.; PEREIRA, C. R. Avaliação da eficiência de horticultores agroecológicos utilizando análise envoltória de dados. Horticultura Brasileira, v. 32, n. 3, p. 336-341, 2014.

OLIVEIRA, E.; SOARES DE MELLO, J. C. C. B.; ASSIS, R. L.; AQUINO, A. M. Análise da eficiência produtiva relacionada com os teores de matéria orgânica no solo, de sistemas de produção orgânicos, em espaço periurbano no município de Nova Iguaçu (RJ). ESPACIOS, v. 38, n. 24, 2017.

PEREIRA, C. N.; SILVEIRA, J. M. F. J. Análise Exploratória da Eficiência Produtiva das Usinas de Cana-de-açúcar na Região Centro-Sul do Brasil. RESR - Economia e Sociologia Rural, v. 54, n. 01, p. 147-166, 2016. http://dx.doi.org/10.1590/1234-56781806-9479005401008

PEREIRA, M. S.; ESPINDOLA, J. A. A.; ASSIS, R. L.; RODRIGUES, G. S.; RODRIGUES, I. A. Avaliação da sustentabilidade de sistemas de produção agrícola de base ecológica no Município de Nova Friburgo, RJ. SBSP Sociedade Brasileira de Sistemas de Produção. Pelotas - RS, 06 a 08 de julho de 2016. Anais, p. 1798 - 1818, 2016.

PLANAPO, Plano nacional de agroecologia e produção orgânica. Brasília, DF: MDS; CIAPO, 2013. Disponível em <http://www.mda.gov.br/sitemda/sites/sitemda/files/user img 19/BrasilAgroecologico_Baixar.pdf>. Acesso em: 21 jan. 2017.

PNA, Programa Nacional de Conservação da Agrobiodiversidade Boletim de avaliação e monitoramento, 2011. Disponível em: <http://terradedireitos.org.br/wpcontent/uploads/2011/04/Programa-Nacional-de-

Conserva\%C3\%A7\%C3\%A3o-da-Agrobiodiversidade-

INTERNET-1.pdf> Acesso em: 21 jan. 2017.

SALGADO JUNIOR, A. P.; CARLUCCI, F. V.; NOVI, J. C Aplicação da análise envoltória de dados (AED) na avaliação da eficiência operacional relativa entre usinas de cana-deaçúcar no território brasileiro. Engenharia Agrícola, Jaboticabal, v. 34, n. 5, p. 826-843, 2014.

SANTOS, J. G.; CÂNDIDO, G. A. Sustentabilidade e agricultura familiar: um estudo de caso em uma associação de agricultores rurais. Revista de Gestão Social e Ambiental, v. 7, n. 1, p. $70-86,2013$
SCHNEIDER, SERGIO. Reflexões sobre diversidade e diversificação. Ruris, v. 4, n. 1, p. 85-131, 2010.

SOARES DE MELLO, J. C. C. B.; MEZA, L. A.; GOMES, E. G.; SERAPIÃO, B. P.; LINS, M. P. E. Análise de envoltória de dados no estudo da eficiência e dos benchmarks para companhias aéreas brasileiras. Pesquisa Operacional, v. 23 , p. 2, p. 325-345, 2003.

WU, J.; LIANG, L.; CHEN, Y. Achievement and benchmarking of countries at the Summer Olympics using cross efficiency evaluation method. European Journal of Operational Research, n. 197, p. 722-730, 2009. 